\title{
The choice number versus the chromatic number for graphs embeddable on orientable surfaces
}

\author{
Niranjan Balachandran Brahadeesh Sankarnarayanan* \\ Department of Mathematics \\ Indian Institute of Technology Bombay \\ Mumbai 400076, Maharashtra, India \\ \{niranj, bs\}@math.iitb.ac.in
}

Submitted: Feb 23, 2021; Accepted: Nov 30, 2021; Published: Dec 17, 2021

(C) The authors. Released under the CC BY-ND license (International 4.0).

\begin{abstract}
We show that for loopless 6-regular triangulations on the torus the gap between the choice number and chromatic number is at most 2 . We also show that the largest gap for graphs embeddable in an orientable surface of genus $g$ is of the order $\Theta(\sqrt{g})$, and moreover for graphs with chromatic number of the order $o\left(\sqrt{g} / \log _{2}(g)\right)$ the largest gap is of the order $o(\sqrt{g})$.
\end{abstract}

Mathematics Subject Classifications: 05C15, 05C10, 05C35, 05C75

\section{Introduction}

We shall denote by $\mathbb{N}$ the set of natural numbers $\{0,1,2, \ldots\}$. For a graph $G=(V, E)$, the notation $v \rightarrow w$ shall indicate that the edge $v \sim w \in E$ is oriented from the vertex $v$ to the vertex $w$. A graph $G$ is directed if every edge of $G$ is oriented. All logarithms in this paper are to the base 2. We freely make use of the Bachmann-Landau-Knuth notations, but state them briefly for completeness below. Let $f$ and $g$ be positive functions of real variables. (1) $f=o(g)$ if $\lim _{x \rightarrow \infty} f(x) / g(x)=0 ;(2) f \leqslant O(g)$ if there is a constant $M>0$ such that $\limsup _{x \rightarrow \infty} f(x) / g(x) \leqslant M$; (3) $f \geqslant \Omega(g)$ if $g \leqslant O(f)$; and $(4) f=\Theta(g)$ if $\Omega(g) \leqslant f \leqslant O(g)$.

A (vertex) coloring of a graph $G=(V, E)$ is an assignment of a "color" to each vertex, that is, an assignment $v \mapsto \operatorname{color}(v) \in \mathbb{N}$ for every $v \in V(G)$. A coloring of $G$ is proper if adjacent vertices receive distinct colors. $G$ is $k$-colorable if there exists a proper coloring of the vertices using at most $k$ colors. The least integer $k$ for which $G$ is $k$-colorable is

*Supported by the National Board for Higher Mathematics (NBHM), Department of Atomic Energy (DAE), Govt. of India. 
called the chromatic number of $G$ and is denoted $\chi(G)$. If $\chi(G)=k$, we also say that $G$ is $k$-chromatic.

A variation of $k$-colorability called $k$-choosability was defined independently by Vizing [33] in 1976 and Erdős, Rubin and Taylor [13] in 1979. A list assignment $\mathcal{L}$ on $G$ is a collection of sets of the form $\mathcal{L}=\left\{L_{v} \subset \mathbb{N}: v \in V(G)\right\}$, where one thinks of each $L_{v}$ as a list of colors available for coloring the vertex $v \in V(G) . G$ is $\mathcal{L}$-choosable if there exists a proper coloring of the vertices such that $\operatorname{color}(v) \in L_{v}$ for every $v \in V(G)$. $G$ is $k$-choosable if it is $\mathcal{L}$-choosable for every list assignment $\mathcal{L}$ with $\left|L_{v}\right| \geqslant k$ for all $v \in V(G)$. The least integer $k$ for which $G$ is $k$-choosable is called the choice number or list chromatic number of $G$ and is denoted $\chi_{\ell}(G)$. If $\chi_{\ell}(G)=k$, we also say that $G$ is $k$-list-chromatic.

The choice number generalizes the chromatic number in the following sense: if $\mathcal{L}$ is a list assignment in which all the lists $L_{v}$ are identical and have cardinality at most $k$, then $G$ is $\mathcal{L}$-choosable if and only if $G$ is $k$-colorable. Hence, $\chi(G) \leqslant \chi_{\ell}(G)$ for any graph $G$. In general, however, $\chi(G)<\chi_{\ell}(G)$, so it behooves one to investigate the nature of the gap between the choice number and chromatic number.

One line of investigation is to examine chromatic-choosable graphs, that is, graphs that satisfy $\chi(G)=\chi_{\ell}(G)$. We mention one of the important results concerning such graphs, conjectured by Ohba [24] in 2002 and subsequently settled in the affirmative by Noel, Reed and $\mathrm{Wu}$ [23] in 2015: if $G$ is a graph on at most $2 \chi(G)+1$ vertices, then it satisfies $\chi(G)=\chi_{\ell}(G)$.

The opposite line of investigation is to examine the width of the gap between the chromatic number and choice number. In [13], Erdôs, Rubin and Taylor showed that there are bipartite graphs (that is, graphs with $\chi(G)=2$ ) that have arbitrarily large choice number; more precisely, they showed that $\chi_{\ell}\left(K_{n, n}\right)>k$ if $n \geqslant\left(\begin{array}{c}2 k-1 \\ k\end{array}\right)$. At first glance it appears that this line of investigation is thus fruitless, but one has to note that the graphs $K_{n, n}$ have high average degree. In fact, Alon [3] showed in 2000 that $\chi_{\ell}(G) \geqslant\left(\frac{1}{2}-o(1)\right) \log (\delta)$, where $\delta \equiv \delta(G)$ is the minimum degree of $G$.

Thus, one is motivated to bound the minimum degree of graphs in order to examine the gap between the chromatic number and choice number. A natural criterion for doing so is to consider graphs that are embeddable in a fixed surface. By a surface we mean a compact connected 2-manifold. Informally, a graph $G=(V, E)$ is embeddable in a surface if there exists a drawing of $G$ on the surface without any crossing edges. By the classification of surfaces theorem, every orientable surface is homeomorphic to a sphere with $g \geqslant 0$ handles, denoted $S_{g}$, and every nonorientable surface is homeomorphic to a sphere with $k \geqslant 1$ crosscaps, denoted $N_{k}$. The genus of the surface $S_{g}$ (resp. $N_{k}$ ) is defined to be $g$ (resp. $k$ ). We shall primarily restrict our attention to graphs embeddable on orientable surfaces in what follows.

Now, suppose that $G=(V, E)$ is a connected graph which is embeddable in $S_{g}, g \geqslant 0$. Choose an embedding and denote by $\mathrm{v}$, e and $f$ the number of vertices, edges and faces of $G$, respectively, in this embedding. Euler's formula says that $\mathrm{v}-\mathrm{e}+\mathrm{f} \geqslant 2-2 g$, with equality holding if every face is homeomorphic to a disc. Let $F \equiv F(G)$ be the set of faces of $G$ in this embedding. If degree $(f) \geqslant 3$ for each $f \in F$, then we have $3 \mathrm{f} \leqslant 2 \mathrm{e}$, and this bounds the minimum degree as $\delta(G) \leqslant 2 \mathrm{e} / \mathrm{v} \leqslant 6+12(g-1) / \mathrm{v}$. Hence, $\delta(G) \leqslant 5$ if 
$g=0$ and $\delta(G) \leqslant 12 g+6$ if $g \geqslant 1$. Thus, we make the following definitions:

Definition 1. For a graph $G=(V, E)$, define the jump of $G$ by jump $(G):=\chi_{\ell}(G)-\chi(G)$. For each $g \geqslant 0$, define the jump at $g$ by

$$
\operatorname{jump}(g):=\max \left\{\operatorname{jump}(G): G \text { is embeddable in } S_{g}\right\} \text {. }
$$

For graphs embeddable in the sphere $S_{0}$ (equivalently, for planar graphs), the investigation of jump(0) was indicated by Erdôs, Rubin and Taylor [13] through the following conjectures and question:

(C1) Every planar graph is 5-choosable.

(C2) There exists a planar graph that is not 4-choosable.

(Q) Does there exist a planar bipartite graph that is not 3-choosable?

Alon and Tarsi [4] in 1992 answered (Q) in the negative by showing that every planar bipartite graph is 3-choosable; this is also best possible since there are simple examples [13] of planar bipartite graphs that are not 2-choosable. Voigt [34] in 1993 settled (C2) positively by constructing a planar graph on 238 vertices that is not 4-choosable, and Thomassen [32] in 1994 settled (C1) positively through a remarkably short and elegant proof.

Thus, jump $(0) \leqslant 2$, with equality holding if and only if there exists a planar 3-chromatic graph that is not 4-choosable. Mirzakhani [19] in 1996 constructed such a graph on 63 vertices; parallelly, Voigt and Wirth [35] in 1997 observed that a non-4-choosable planar graph on 75 vertices constructed by Gutner [15] in 1996 is 3-chromatic. Thus, it is known that $\operatorname{jump}(0)=2$.

We pose the analogous question for toroidal graphs:

Question 2. What is jump(1)? That is, how large can the gap between the choice number and chromatic number be for a toroidal graph?

Since every planar graph is also toroidal, the maximum gap for toroidal graphs cannot be smaller than 2. In Section 3, we examine 6-regular triangulations on the torus and show the following result:

Theorem 3. For any loopless 6 -regular triangulation $G$ on the torus, jump $(G) \leqslant 2$.

While computing jump $(g)$ precisely for larger values of $g$ seems difficult, we are able to describe the asymptotic behavior of jump $(g)$ in Section 4 as follows:

Theorem 4. jump $(g)=\Theta(\sqrt{g})$. That is, there exist two positive constants $c_{1}$ and $c_{2}$ such that

$$
c_{1} \sqrt{g} \leqslant \operatorname{jump}(g) \leqslant c_{2} \sqrt{g}
$$

for all sufficiently large $g$. 
A natural follow-up is to investigate for which graphs this largest gap is attained, for which the following classical result is useful:

Theorem 5 (Heawood [16], 1890). Let $g \geqslant 1$. If $G$ is embeddable in $S_{g}$, then

$$
\chi(G) \leqslant \chi_{\ell}(G) \leqslant H(g):=\left\lfloor\frac{7+\sqrt{1+48 g}}{2}\right\rfloor .
$$

Heawood proved that $H(g)$, the so called Heawood number, is an upper bound for $\chi(G)$, and essentially the same argument carries forward to prove that $H(g)$ is an upper bound for $\chi_{\ell}(G)$ as well. Now, Theorem 5 shows that if $G$ is $H(g)$-chromatic then it is also $H(g)$-list-chromatic, so such graphs can never attain the maximum gap for $S_{g}$. The same is true if $G$ is $(H(g)-1)$-chromatic, by the following result known as Dirac's map color theorem:

Theorem 6 (Dirac [11], 1952, Böhme-Mohar-Stiebitz [9], 1999). If G is embeddable in $S_{g}$ with $\chi(G)=H(g)$ or $\chi_{\ell}(G)=H(g)$, then $K_{H(g)}$ is a subgraph of $G$.

Dirac proved this result for the chromatic number, and it was later extended to the choice number by Böhme, Mohar and Stiebitz.

At the other end, consider the complete bipartite graph $K_{n, n}$. It is an easy exercise that $K_{n, n}$ is $k$-choosable where $k:=\lfloor\log (n)\rfloor+1$, and it is known that $K_{m, n}$ is embeddable in $S_{g}$ for $g:=\lceil(m-2)(n-2) / 4\rceil$ and this is best possible (see [27]). Hence,

$$
\operatorname{jump}\left(K_{n, n}\right) \leqslant \log (n)-1 \leqslant \log (2 \sqrt{g+1}+2)-1 \leqslant O(\log (g)) \leqslant o(\sqrt{g}) .
$$

Since any bipartite graph is a subgraph of a complete bipartite graph, this shows that one does not expect bipartite graphs to attain the greatest gap on a fixed surface.

This motivates the following definition:

Definition 7. For each $g \geqslant 0, r \geqslant 1$, define

$$
\operatorname{jump}(g, r):=\max \left\{\operatorname{jump}(G): G \text { is connected and embeddable in } S_{g}, \chi(G)=r\right\}
$$

whenever the set on the right is nonempty. If there is no connected graph embeddable in $S_{g}$ having chromatic number $r$, then define jump $(g, r):=0$.

In Section 4, we prove the following stronger result along the same lines as the bipartite case:

Theorem 8. jump $(g, r)=o(\sqrt{g})$ when $r=o(\sqrt{g} / \log (g))$. That is, if for each $\delta>0$ we have $r \leqslant \delta \sqrt{g} / \log (g)$ for all sufficiently large $g$, then for every $\epsilon>0$, jump $(g, r) \leqslant \epsilon \sqrt{g}$ for all sufficiently large $g$.

The rest of the paper is organised as follows. In Section 2, we state some preliminary results that will be used in our proofs later on. In Section 3, we prove Theorem 3. In Section 4, we prove Theorems 4 and 8. In Section 5, we mention some partial results towards computing jump(1), that is, the maximum gap $\chi_{\ell}(G)-\chi(G)$ for toroidal $G$. We also generalise the definitions of $\operatorname{jump}(g)$ and $\operatorname{jump}(g, r)$, as well as the results proved in Section 4, to graphs embeddable on nonorientable surfaces. We conclude the paper with some open questions for further investigation. 


\section{Preliminaries}

We begin with the following result on the embeddability of the complete graph $K_{r}$ into an orientable surface:

Theorem 9 (Ringel-Youngs [28], 1968). For every $r \geqslant 1, K_{r}$ is embeddable in $S_{\gamma(r)}$ for $\gamma(r)=\lceil(r-3)(r-4) / 12\rceil$, and this is best possible.

The above result, called the Ringel-Youngs theorem, also shows that $K_{H(g)}$ is embeddable in $S_{g}$ for all $g \geqslant 1$, so it implies that Heawood's upper bound is tight for all $g \geqslant 1$.

Definition 10. Let $G=(V, E)$ be a simple graph, and $k \geqslant 1$. The $k$-core of $G$ is the unique maximal subgraph of $G$ having minimum degree at least $k$.

The next result is folklore; it was observed for $k=2$ by Erdős, Rubin and Taylor [13], but we include the proof for general $k$ for the sake of completeness.

Proposition 11. Let $G=(V, E)$ be a simple graph, and $k \geqslant 1$. Then, $G$ is $k$-choosable if and only if its $k$-core is $k$-choosable.

Proof. If $G$ is $k$-choosable, then so is every subgraph. In particular, its $k$-core is $k$ choosable. This proves the forward direction. For the reverse direction, suppose for the sake of contradiction that the $k$-core of $G$ is $k$-choosable, but $G$ is not $k$-choosable. Let $H \leqslant G$ be a subgraph of $G$ with the least order such that $H$ is not $k$-choosable. Note that $H$ must be nonempty.

We show that the minimum degree of $H$ must be at least $k$. Suppose for the sake of contradiction that there exists a vertex $v \in V(H)$ such that degree $(v)<k$. Let $\mathcal{L}$ be a list assignment on $G$ with lists of size $k$ for which $H$ is not $\mathcal{L}$-choosable. The subgraph $H-v$ is $k$-choosable by the minimality of $H$ with respect to the number of vertices, so properly color $H-v$ using the list assignment $\mathcal{L}$. Now, observe that the list $L_{v}$ on the vertex $v$ has lost at most $k-1$ colors due to the coloring of its neighbors, hence the coloring on $H-v$ extends to a proper coloring on $H$. Hence, $H$ is $\mathcal{L}$-choosable, a contradiction.

Thus, the minimum degree of $H$ is at least $k$, and so $H$ is a subgraph of the $k$-core of $G$. But the $k$-core of $G$ is $k$-choosable by assumption, so $H$ is also $k$-choosable, a contradiction. This completes the proof.

The next result, called Brooks's theorem, gives a useful bound on the chromatic and choice numbers of a graph in terms of its maximum degree.

Theorem 12 (Brooks [10], 1941, Vizing [33], 1976, Erdős-Rubin-Taylor [13], 1979). Let $G$ be a connected simple graph with maximum degree $\Delta$. Then, $G$ is $\Delta$-colorable (resp. $\Delta$-choosable), unless $G$ is an odd cycle or the complete graph on $\Delta+1$ vertices, in which cases $G$ is $(\Delta+1)$-chromatic (resp. $(\Delta+1)$-list-chromatic).

Brooks proved his result for the chromatic number, and this was later extended to the choice number independently by Vizing and by Erdős, Rubin and Taylor.

Another useful result in computing the choice number of graphs is as follows. 
Definition 13. For a directed graph $G$, a subgraph $H$ is Eulerian if $\operatorname{indegree}_{H}(v)=$ outdegree $_{H}(v)$ for all $v \in V(H)$. An even (resp. odd) Eulerian subgraph is one with an even (resp. odd) number of edges.

Note that the subgraphs are not assumed to be connected in the above definition.

Theorem 14 (Alon-Tarsi [4], 1992). Suppose the edges of a graph $G$ can be oriented such that the number of even Eulerian subgraphs differs from the number of odd Eulerian subgraphs. Let $\mathcal{L}$ be a list assignment such that $\left|L_{v}\right| \geqslant$ outdegree $(v)+1$ for all $v \in V(G)$. Then, $G$ is $\mathcal{L}$-choosable.

Notation. For every $m, r \geqslant 1$, denote by $K_{m * r}$ the complete $r$-partite graph with $m$ vertices in each part.

We will need the following bound on the growth of the choice number of $K_{m * r}$ :

Theorem 15 (Alon [2], 1992). There exist two positive constants $c_{1}$ and $c_{2}$ such that, for every $m \geqslant 2$ and for every $r \geqslant 2$,

$$
c_{1} r \log (m) \leqslant \chi_{\ell}\left(K_{m * r}\right) \leqslant c_{2} r \log (m) .
$$

If $G=(V, E)$ is a $k$-regular triangulation on the torus $S_{1}$, then $k \mathrm{v}=2 \mathrm{e}$ and $3 \mathrm{f}=2 \mathrm{e}$, which together with Euler's formula $\mathrm{v}-\mathrm{e}+\mathrm{f}=0$ for $S_{1}$ gives $k=6$. So, any regular triangulation on the torus is necessarily 6-regular. In 1973, Altshuler [5] characterized the 6-regular triangulations on the torus as follows (similar constructions can also be found in $[21,30])$. For integers $r \geqslant 1, s \geqslant 1$, and $0 \leqslant t<s$, let the graph $G:=T(r, s, t)$ have the vertex set $V(G):=\{(i, j): 1 \leqslant i \leqslant r, 1 \leqslant j \leqslant s\}$ and the following edges:

- For $1<i<r$, the vertex $(i, j)$ is adjacent to $(i, j \pm 1),(i \pm 1, j)$ and $(i \pm 1, j \mp 1)$.

- If $r>1$, the vertex $(1, j)$ is adjacent to $(1, j \pm 1),(2, j),(2, j-1),(r, j+t+1)$ and $(r, j+t)$.

- If $r>1$, the vertex $(r, j)$ is adjacent to $(r, j \pm 1),(r-1, j+1),(r-1, j),(1, j-t)$ and $(1, j-t-1)$.

- If $r=1$, the vertex $(1, j)$ is adjacent to $(1, j \pm 1),(1, j \pm t)$ and $(1, j \pm(t+1))$.

Here, addition in the second coordinate is taken modulo $s$.

$T(r, s, t)$ can be visualized by taking an $(r+1) \times(s+1)$ grid graph, identifying the top and bottom rows in the usual manner, identifying the leftmost and rightmost columns with a "twist" by $t$ vertices, and then triangulating each face in a regular manner. Figure 1 shows the graph $G=T(5,6,2)$. The edges between the top and bottom rows are not shown in the figure. Note that the graph in Figure 1 happens to be 3-chromatic.

Theorem 16 (Altshuler [5], 1973). Every 6-regular triangulation on the torus is isomorphic to $T(r, s, t)$ for some integers $r \geqslant 1, s \geqslant 1$, and $0 \leqslant t<s$.

Note that $T(r, s, t)$ can be isomorphic to $T\left(r^{\prime}, s^{\prime}, t^{\prime}\right)$ even if the tuples $(r, s, t)$ and $\left(r^{\prime}, s^{\prime}, t^{\prime}\right)$ are distinct. 


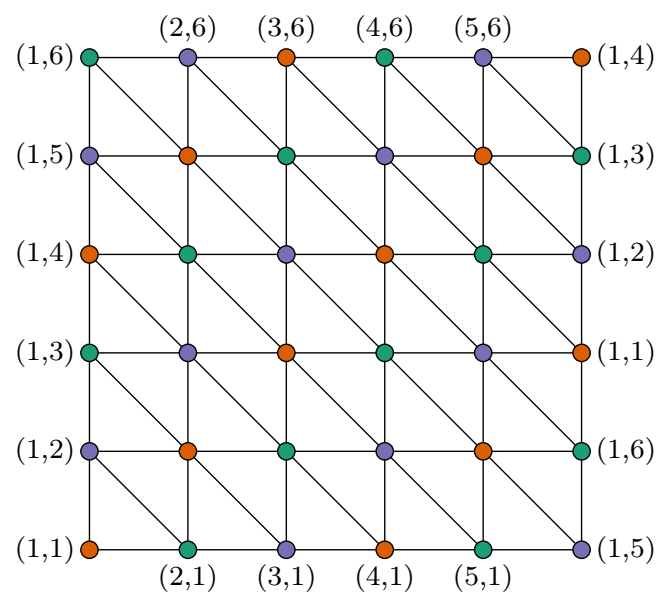

Figure 1: $G=T(5,6,2)$

\section{The gap for 6-regular triangulations on the torus}

We start by observing that some of the graphs $T(r, s, t)$ are not simple triangulations: they may contain loops and multiple edges.

The graphs containing loops are precisely those isomorphic to $T(1, s, 0)$ for all $s \geqslant 1$, so we do not consider them. The loopless graphs containing multiple edges are precisely those isomorphic to $T(2, s, t)$ for $t=0, s-2, s-1, s \geqslant 2$, and $T(1, s, t)$ for $t=1,\lfloor(s-1) / 2\rfloor$, $s \geqslant 3$. One can check that these graphs are never bipartite, so the chromatic number of any such graph is at least 3 . Moreover, after deleting the duplicate edges (since they do not make a difference for the purpose of coloring) these graphs have maximum degree $\Delta \leqslant 5$. Hence, by Theorem 12 , any such graph is 5 -choosable and thus has gap at most 2 , unless it is isomorphic to $K_{6}$; but in the latter case, Theorem 12 says that the chromatic number and choice number are both equal to 6 , so the gap is 0 . Hence, jump $(T(r, s, t)) \leqslant 2$ if $T(r, s, t)$ contains multiple edges.

This takes care of the trivial cases. Next, we examine the graphs $T(r, s, t)$ that are simple. First, we establish some notations and definitions for use in the upcoming proofs:

Notation.

- A list $L_{v}$ is called a $k$-list if $\left|L_{v}\right|=k$. A $k$-list assignment $\mathcal{L}$ on a graph $G$ is a list assignment for which every list $L_{v}$ is a $k$-list. Note that a graph $G$ is $k$-choosable if and only if it is $\mathcal{L}$-choosable for every $k$-list assignment $\mathcal{L}$.

- Let $G$ be a graph with a list assignment $\mathcal{L}$. For a subgraph $G^{\prime}$ of $G$ and a color $c \in \mathbb{N}$, denote by $G^{\prime}(c)$ the induced subgraph of $G^{\prime}$ on those vertices whose lists contain the color $c$. We shall denote (maximal connected) components of $G^{\prime}(c)$ by $\alpha, \beta$, etc.

- Let $P$ be a nonempty path or cycle graph. A vertex $v \in V(P)$ is an end point of $P$ if degree $(v)=1$, and it is an interior point of $P$ if $\operatorname{degree}(v)=2$. 
- Let $P^{\prime}$ be a nonempty proper connected subgraph of $P$ (so $P^{\prime}$ is itself a nonempty path graph). We denote by $v\left(P^{\prime}\right)$ an end point of $P^{\prime}$, and by $w\left(P^{\prime}\right)$ a vertex in $P \backslash P^{\prime}$ that is adjacent to $v\left(P^{\prime}\right)$ (when it exists).

Next, we record the following simple observation:

Observation 17. Suppose $P$ is a nonempty path or cycle graph, $\mathcal{L}$ is a $k$-list assignment on $P(k \geqslant 1)$, and $c \in \mathbb{N}$ is any color such that $P(c)$ is a nonempty proper subgraph of $P$. Let $\alpha$ be a component of $P(c)$. Let $v(\alpha)$ be an end point of $\alpha$ for which $w(\alpha)$ exists. Then, there exists a color $d \in L_{w(\alpha)} \backslash L_{v(\alpha)}$, since $c \notin L_{w(\alpha)}$ and $\left|L_{v(\alpha)}\right|=\left|L_{w(\alpha)}\right|=k \geqslant 1$. In particular, there exists a component $\beta$ of $P(d)$ containing $w(\alpha)$ but not $v(\alpha)$.

The following key lemma will allow us to color the alternate vertices of a pathequipped with a list assignment - in such a way that the remaining vertices lose at most one color from each of their lists.

Lemma 18. Let $P=(V, E)$ be a path graph on at least 2 vertices, and $\mathcal{L}$ a $k$-list assignment on $P, k \geqslant 1$. Let $I_{1} \cup I_{2}$ be the unique partition of $V(P)$ into two independent sets. Then, there exists a coloring of $I_{1}$ such that every $v \in I_{2}$ loses at most one color from its list.

Proof. Fix an end point $v(P)$ of $P$. Choose a color $c \in L_{v(P)}$ and consider the component $\alpha$ of $P(c)$ containing $v(P)$. Set color $(v)=c$ for every $v \in I_{1} \cap V(\alpha)$.

If $\alpha=P$, then we are done, since every $v \in I_{2}$ has lost at most one color from its list, namely $c$. So, suppose that $\alpha \neq P$, and define $P_{1}:=P-\alpha$. Then, $P_{1}$ is a nonempty path, and there is an end point $v(\alpha)$ of $\alpha$ such that $w(\alpha)$ is an end point of $P_{1}$. Choose $c_{1} \in L_{w(\alpha)} \backslash L_{v(\alpha)}$, which exists by Observation 17. Let $\alpha_{1}$ be the component of $P_{1}\left(c_{1}\right)$ containing $w(\alpha)$, and set $\operatorname{color}(v)=c_{1}$ for every $v \in I_{1} \cap V\left(\alpha_{1}\right)$.

Again, if $\alpha_{1}=P_{1}$, then we are done. Else, let $P_{2}:=P_{1} \backslash \alpha_{1}$, and proceed inductively until the process terminates.

\section{Proof of Theorem 3}

Proof. Let us first show that any simple 6-regular toroidal triangulation $G$ that is 3chromatic is 5 -choosable.

By Theorem 16, $G$ is isomorphic to $T(r, s, t)$ for some integers $r \geqslant 1, s \geqslant 1$, and $0 \leqslant t<s$. It is straightforward to check that when $T(r, s, t)$ is a simple graph, it is 3 -chromatic if and only if $s \equiv 0 \equiv r-t(\bmod 3)$. Moreover, $T(r, s, t)$ is uniquely 3colorable whenever this happens, so let $I_{1}, I_{2}, I_{3}$ be the three independent sets defined by any coloring of $T(r, s, t)$ with 3 colors. Without loss of generality, we fix $I_{j}$ to be the independent set containing $(1, j)$ for $j=1,2,3$.

Consider the subgraph $G_{1}:=G-I_{1}$ of $G$. Note that $G_{1}$ is a 3-regular bipartite graph. Orient the edges of $G_{1}$ as follows: every horizontal edge is directed east, every vertical edge is directed north, and every diagonal edge is directed south-east. (Figure 2 shows this for $G=T(5,6,2)$.) More formally, give the orientations as follows (recall that addition in the second coordinate is taken modulo $s$ ). For every $1 \leqslant i \leqslant r$ and $1 \leqslant j \leqslant s$ such that $j-i \not \equiv 0(\bmod 3)$ : 


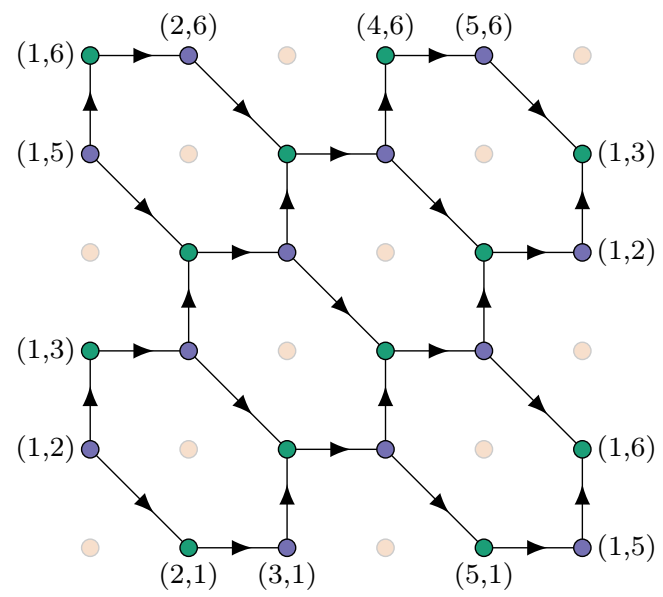

Figure 2: $G_{1}$ with oriented edges

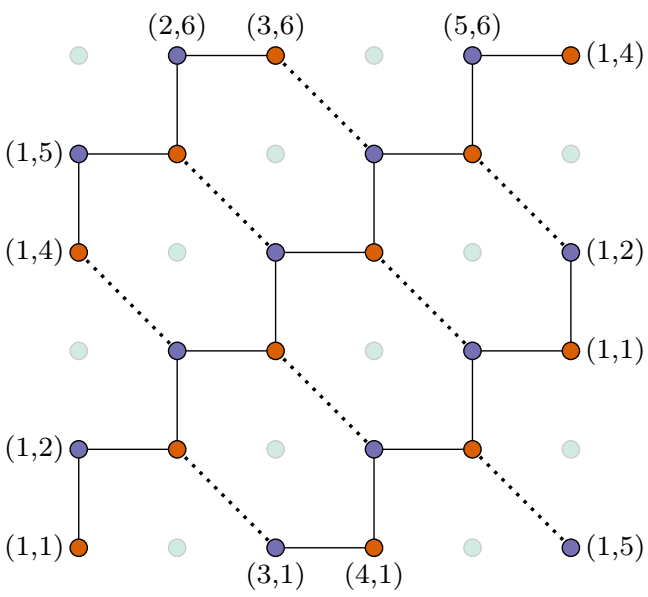

Figure 3: The subgraph $H$

- if $j-i \equiv 2(\bmod 3), \operatorname{assign}(i, j) \rightarrow(i+1, j)$ for all $1 \leqslant i<r$, and $(r, j) \rightarrow(1, j-t)$;

- if $j-i \equiv 1(\bmod 3)$, assign $(i, j) \rightarrow(i, j+1)$ for all $1 \leqslant i \leqslant r,(i, j) \rightarrow(i+1, j-1)$ for all $1 \leqslant i<r$, and $(r, j) \rightarrow(1, j-t-1)$.

Then, outdegree $G_{1}(v)=2$ for every $v \in I_{2}$, and outdegree $G_{1}(v)=1$ for every $v \in I_{3}$.

We claim that $G_{1}$ with this orientation has no odd Eulerian subgraphs. For, if $H$ is an Eulerian subgraph of $G_{1}$ and $v \in V(H)$ is not an isolated vertex, then $\operatorname{degree}_{H}(v)$ is a positive even integer, so degree ${ }_{H}(v)=2$. Thus, $H$ is a disjoint union of cycles and isolated vertices, but every cycle in a bipartite graph is even, so this proves our claim. Since the empty graph is an even Eulerian subgraph of $G_{1}$, by Theorem $14 G_{1}$ is $\mathcal{L}$-choosable for every list assignment $\mathcal{L}$ such that $\left|L_{v}\right| \geqslant 3$ for all $v \in I_{2}$ and $\left|L_{v}\right| \geqslant 2$ for all $v \in I_{3}$.

Now, suppose that we are given a 5 -list assignment $\mathcal{L}$ on $G$. If we arbitrarily assign $\operatorname{color}(v) \in L_{v}$ for every $v \in I_{1}$, then -in the worst-case scenario-we are left with a 2-list assignment on $G_{1}=G-I_{1}$. However, to apply Theorem 14 on $G_{1}$ with the orientation described above, we need 3-lists on $I_{2}$. So, let us now consider the subgraph $H$ on $I_{1} \cup I_{2}$ with only the horizontal and vertical edges present (see Figure 3). We will show that we can color $I_{1}$ carefully in such a way that every vertex in $I_{2}$ loses at most one color in $H$; thus every vertex in $I_{2}$ will lose at most two colors in $G$ (since every $v \in I_{2}$ is adjacent to one more vertex of $I_{1}$ in $G$ than in $H$, shown by the dotted edges in Figure 3$)$. Then, we will be done by invoking Theorem 14 .

Note that $H$ is a disjoint union of even cycles (in fact, there are exactly $\operatorname{gcd}(s, r-t) / 3$ cycles in $H$ ). First, assume for the sake of simplicity that there is only one cycle in $H$ (as in Figure 3). We eliminate the trivial case: if there is a color that belongs to every list on $I_{1}$, then we just color $I_{1}$ with that color, and we are done. Thus, it suffices to assume that there is no color common to every list on $I_{1}$.

Now, suppose there exists a color $c$ such that $H(c)$ has a component $\alpha$ of even order. Assign $\operatorname{color}(v)=c$ for all $v \in I_{1} \cap V(\alpha)$. Observe that:

- Every $v \in V(\alpha) \cap I_{2}$ loses at most one color (namely, $c$ ) from its list. 
- Since $\alpha$ has even order, it has endpoints $v_{j}(\alpha)$ in $I_{j}$ for $j=1,2$.

- The vertex $w_{1}(\alpha) \in I_{2}$ has not lost any color in its list due to the coloring of $v_{1}(\alpha)$, so we are free to put any color on the other vertex adjacent to $w_{1}(\alpha)$.

- On the other hand, the vertex $w_{2}(\alpha) \in I_{1}$ must be colored carefully, since $v_{2}(\alpha) \in I_{2}$ has already lost one color from its list.

By Observation 17, there exists a color $d \in L_{w_{2}(\alpha)} \backslash L_{v_{2}(\alpha)}$. So, consider the path $P:=$ $H-\alpha$. Apply Lemma 18 to $P$ by starting the coloring at $w_{2}(\alpha)$ with the color $d$. Then, every $v \in I_{2}$ will indeed have lost at most one color from its list, so we are done.

On the other hand, suppose that for every color $c$, every component of $H(c)$ has odd order. Choose a color $c$ and a component $\alpha$ of $H(c)$ such that both ends of $\alpha$ lie in $I_{1}$. We can always do this because if $\alpha^{\prime}$ is a component of $H\left(c^{\prime}\right)$ such that both ends of $\alpha^{\prime}$ lie in $I_{2}$, then choose $c \in L_{w\left(\alpha^{\prime}\right)} \backslash L_{v\left(\alpha^{\prime}\right)}$ for some end point $v\left(\alpha^{\prime}\right)$, and let $\alpha$ be the component of $H(c)$ containing $w\left(\alpha^{\prime}\right)$. Then, both ends of $\alpha$ lie in $I_{1}$ as required. Assign $\operatorname{color}(v)=c$ for all $v \in I_{1} \cap V(\alpha)$. In this case, we have that:

- Every $v \in I_{2} \cap V(\alpha)$ loses at most one color (namely, $c$ ) from its list.

- Since both ends, $v_{1}(\alpha)$ as well as $v_{2}(\alpha)$, lie in $I_{1}$, the vertices $w_{1}(\alpha)$ and $w_{2}(\alpha)$ in $I_{2}$ have not yet lost any color from their lists, so we are free to put any color on their other neighbors.

Thus, we consider the path $P:=H-\alpha$, and apply Lemma 18 to $P$ starting from any end point with any color. Then, every $v \in I_{2}$ will again have lost at most one color from its list, so we are done.

If $H$ consists of more than one cycle, we repeat this process for each cycle. Thus, with this coloring scheme for $I_{1}$, we are left with the required list sizes on the vertices of $G_{1}=G-I_{1}$, so we are done by Theorem 14 . Thus, we have shown that every simple 6-regular toroidal triangulation that is 3-chromatic is 5-choosable.

Now, on the one hand, any simple triangulation requires at least 3 colors for a proper coloring. On the other hand, $H(1)=7$, so every toroidal graph requires no more than 7 colors for a proper coloring by Theorem 5. Furthermore, Theorem 6 shows that $\chi(G)=$ $7 \Longleftrightarrow \chi_{\ell}(G)=7$ for any toroidal graph $G$. Hence, if $G$ is a simple triangulation on the torus with $\operatorname{jump}(G)>2$, then it must be that $\chi(G)=3$ and $\chi_{\ell}(G)=6$. However, we have shown above that this cannot happen if $G$ is 6 -regular. Hence, jump $(G) \leqslant 2$ for any simple 6-regular triangulation $G$ on the torus. We have also seen that jump $(G) \leqslant 2$ for any loopless 6 -regular triangulation $G$ that contains multiple edges. This completes the proof of Theorem 3.

\section{Asymptotics of the jump function}

For the sake of simplicity, we ignore any ceilings and floors in the following proofs. 


\section{Proof of Theorem 4}

Proof. Let $g \geqslant 1$. For any graph $G, \chi_{\ell}(G)-\chi(G) \leqslant \chi_{\ell}(G)$, and if $G$ is embeddable in $S_{g}$ then $\chi_{\ell}(G) \leqslant H(g)$ by Theorem 5 . Thus, jump $(g) \leqslant H(g) \leqslant 7 \sqrt{g}$ for all $g \geqslant 1$.

To establish the lower bound, let $m \geqslant 2$ be fixed, and consider the graph $K_{m * r}$ for $r \geqslant 2$. Note that $\chi\left(K_{m * r}\right)=\chi\left(K_{r}\right)=r$, and $\chi_{\ell}\left(K_{m * r}\right) \geqslant c_{1} r \log (m)$ by Theorem 15 . Hence, jump $\left(K_{m * r}\right) \geqslant c_{1} r \log (m)-r$. Pick $m$ large enough so that $c_{1} \log (m) \geqslant 2$. Then, jump $\left(K_{m * r}\right) \geqslant r$. Thus, we would like to show that $K_{m * r}$ is embeddable in $S_{g}$ for $g \leqslant$ $O\left(r^{2}\right)$.

Theorem 9 says that $K_{r}$ is embeddable in $S_{\gamma(r)}$, so start with an embedding of $K_{r}$ into $S_{\gamma(r)}$ and then add handles to $S_{\gamma(r)}$ to accommodate the extra edges of $K_{m * r}$. Since $\left|E\left(K_{r}\right)\right|=\left(\begin{array}{l}r \\ 2\end{array}\right)$ and $\left|E\left(K_{m * r}\right)\right|=m^{2}\left(\begin{array}{l}r \\ 2\end{array}\right)$, we need to add at most $\left(m^{2}-1\right)\left(\begin{array}{l}r \\ 2\end{array}\right)$ handles to $S_{\gamma(r)}$. Since $\gamma(r)+\left(m^{2}-1\right)\left(\begin{array}{l}r \\ 2\end{array}\right) \leqslant m^{2} r^{2} / 2, K_{m * r}$ is embeddable in $S_{g(r)}$ where $g(r):=m^{2} r^{2} / 2$, and this is what we wanted to show. Hence, jump $(g(r)) \geqslant r=c \sqrt{g(r)}$ for all $r \geqslant 2$, where $c:=\sqrt{2} / m$.

Finally, suppose that $g(r) \leqslant g^{\prime} \leqslant g(r+1)$ for a fixed $r \geqslant 2$. $K_{m * r}$ is embeddable in $S_{g^{\prime}}$ as well, so

$$
\operatorname{jump}\left(g^{\prime}\right) \geqslant \operatorname{jump}\left(K_{m * r}\right) \geqslant r=c \sqrt{g(r+1)}-1 \geqslant c \sqrt{g^{\prime}}-1 \geqslant c^{\prime} \sqrt{g^{\prime}}
$$

for any positive constant $c^{\prime}<c$, provided $r$ (and hence $g(r)$ ) is sufficiently large. This completes the proof. Additionally, we note that the proof of Theorem 15 in [2] shows that one can take $c^{\prime}=2 \times 10^{-113}$.

\section{Proof of Theorem 8}

Proof. Let $\epsilon>0$ and $g \geqslant 1$. Let $G$ be simple and embeddable in $S_{g}$ with $\chi(G)=r$, and let $|V(G)|=m$. To show that $\operatorname{jump}(G) \leqslant \epsilon \sqrt{g}$ it suffices to show that $\chi_{\ell}(G) \leqslant \epsilon \sqrt{g}$ since $\operatorname{jump}(G) \leqslant \chi_{\ell}(G)$. By Proposition 11, there is no loss of generality in assuming that $G$ is equal to its $(\epsilon \sqrt{g})$-core. Thus, the minimum degree, and hence average degree $2 \mathrm{e} / \mathrm{v}$, of $G$ is bounded below by $\epsilon \sqrt{g}$. By Euler's formula, $2 \mathrm{e} / \mathrm{v} \leqslant 6+12(g-1) / m$, and hence $m \leqslant 24 \sqrt{g} / \epsilon$.

Now, $G$ is a subgraph of $K_{m * r}$, and $\chi_{\ell}(G) \leqslant \chi_{\ell}\left(K_{m * r}\right) \leqslant c_{2} r \log (m)$ by Theorem 15 . Then, for every $\delta>0$,

$$
\begin{aligned}
\chi_{\ell}\left(K_{m * r}\right) & \leqslant c_{2} r \log (m) \\
& \leqslant c_{2}\left(\frac{\delta \sqrt{g}}{\log (g)}\right)\left(\log (24 / \epsilon)+\frac{1}{2} \log (g)\right) \\
& \leqslant c_{2} \delta \sqrt{g}\left(\frac{\log (24 / \epsilon)}{\log (g)}+\frac{1}{2}\right) \\
& <c_{2} \delta \sqrt{g}
\end{aligned}
$$

for all sufficiently large $g$. Let $\delta=\epsilon / c_{2}$ to finish the proof. 


\section{Conclusions and further remarks}

\subsection{The jump for general toroidal graphs}

We discuss some partial results towards computing jump $(G)$ for a general toroidal graph $G$.

Firstly, we will need the following result of Alon and Tarsi [4] used in their proof that bipartite planar graphs are 3-choosable. For a graph $G$, define

$$
L(G):=\max _{H \leqslant G}\left\{\frac{|E(H)|}{|V(H)|}\right\},
$$

where the maximum is taken over all subgraphs $H=(V, E)$ of $G$.

Theorem 19 (Alon-Tarsi [4], 1992). Every bipartite graph $G$ is $(\lceil L(G)\rceil+1)$-choosable.

Using Euler's formula, it follows that any planar bipartite graph $G=(V, E)$ satisfies $\mathrm{e} \leqslant 2 \mathrm{v}-4$, so $L(G) \leqslant 2$. Hence, every bipartite planar graph is 3 -choosable.

The same analysis goes through for toroidal bipartite graphs, since Euler's formula applied on toroidal bipartite graphs $G=(V, E)$ yields e $\leqslant 2 \mathrm{v}$, so again $L(G) \leqslant 2$. Thus, every bipartite toroidal graph is also 3-choosable. Hence, if $G$ is a toroidal graph with $\operatorname{jump}(G)>2$, then $G$ cannot be bipartite, so it must be that $\chi(G) \geqslant 3$. On the other hand, if $\chi(G) \geqslant 4$, then jump $(G) \leqslant 2$, since every toroidal graph is 7-choosable, but every 7 -list-chromatic toroidal graph is also 7-chromatic, by Theorem 6 .

Hence, any counterexample to the claim that jump $(1)=2$ must be a toroidal graph $G$ for which $\chi(G)=3$ and $\chi_{\ell}(G)=6$. Now, suppose that there do exist such graphs, and choose one with the minimal number of vertices. Then, by Proposition 11, its minimum degree is at least 5 . Recall that Euler's formula shows that the average degree, $2 \mathrm{e} / \mathrm{v}$, of a toroidal graph $G$ satisfies $2 \mathrm{e} / \mathrm{v} \leqslant 6$, and equality holds if $G$ is a triangulation. Thus, if $\delta(G)=6$, then $G$ is in fact a 6 -regular triangulation. This motivates one to examine 6-regular triangulations in particular, and we have shown in Theorem 3 that jump $(G) \leqslant 2$ for such graphs.

Thus, any minimal counterexample $G$ must satisfy $\delta(G)=5$. Moreover, if we add edges to $G$ while preserving its toroidicity and chromatic number, then we can get a minimal counterexample whose faces are all either triangular or quadrangular, which we call a mosaic following Nakamoto, Noguchi and Ozeki [20]. Thus, it follows that jump(1) > 2 if and only if there is a 3-chromatic toroidal mosaic of minimum degree 5 that is not 5-choosable.

Now, it so happens that there do exist 3-chromatic toroidal graphs $G$ with $\delta(G)=5$ (see Figures 4, 5, and 6; again, the edges between the top and bottom rows are omitted). While ad hoc arguments can show that $\operatorname{jump}(G) \leqslant 2$ for some of these graphs, a unified argument is still missing. An Alon-Tarsi type argument appears more difficult to implement since there may be several vertices with degree greater than 10 , so it is not clear how to orient the edges appropriately.

A relevant concept to mention here is color criticality of graphs. For $k \geqslant 1$, a $k$-critical graph is one that is not $(k-1)$-colorable but whose proper subgraphs are. Similarly, a 


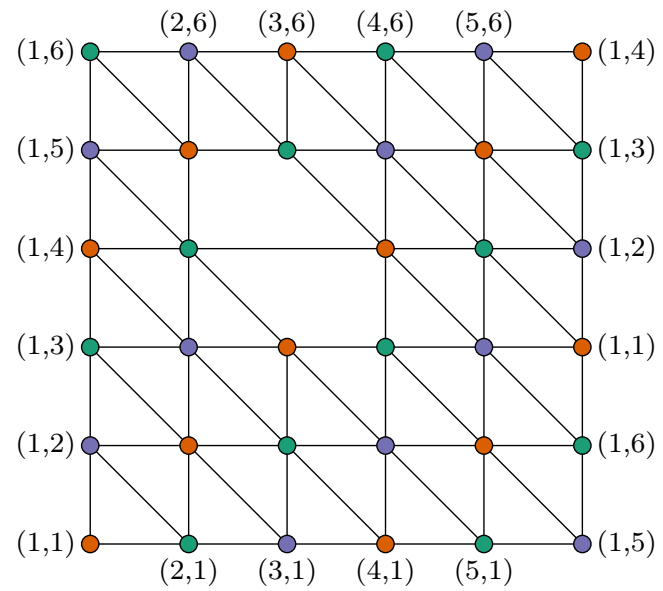

Figure 4: Toroidal $G$ with $\chi(G)=3$, $\delta(G)=5, \Delta(G)=6$

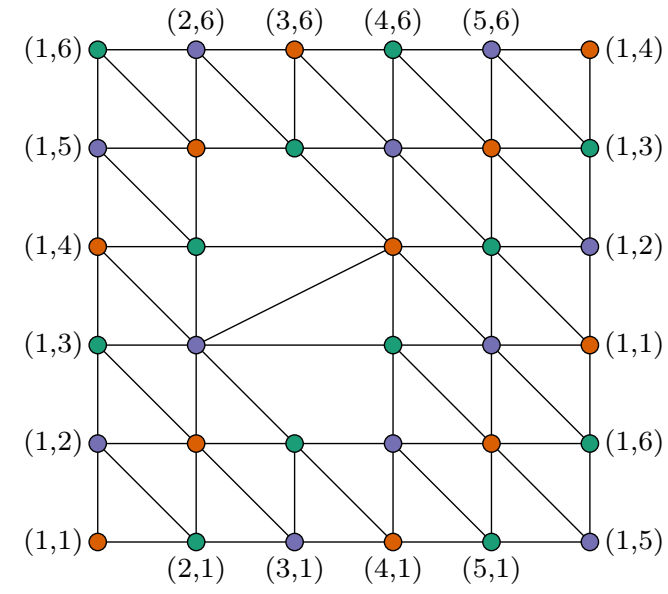

Figure 5: Toroidal $G$ with $\chi(G)=3$, $\delta(G)=5, \Delta(G)=7$

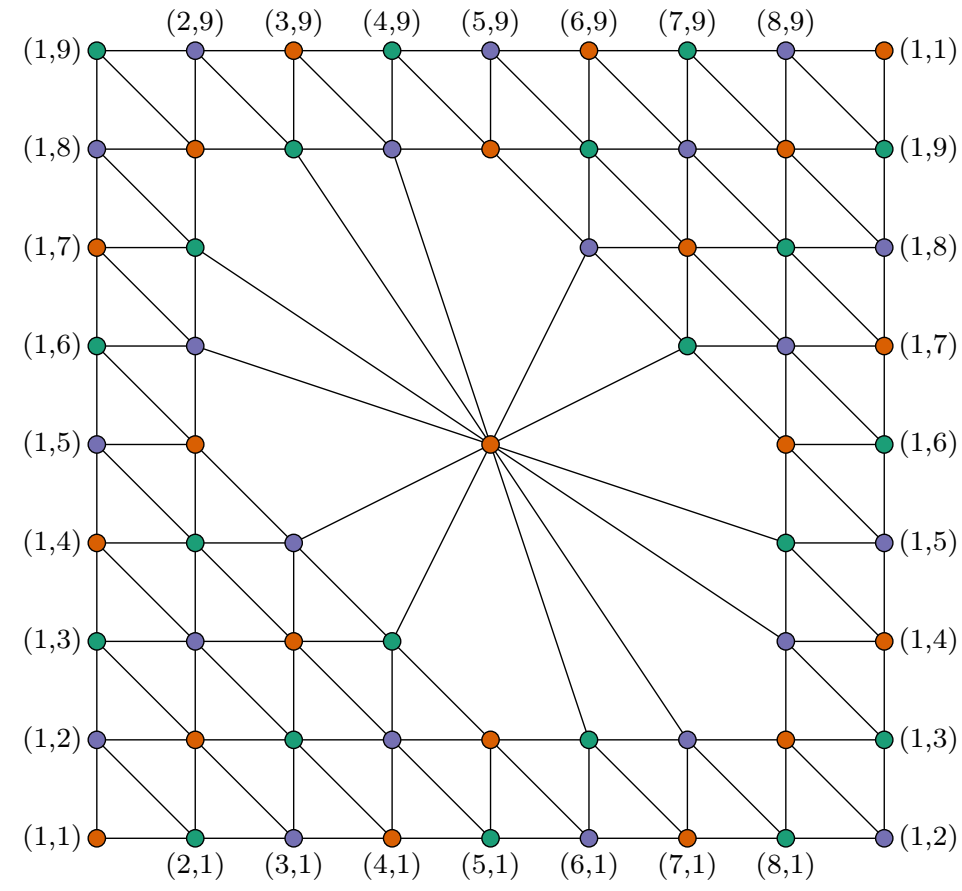

Figure 6: Toroidal $G$ with $\chi(G)=3, \delta(G)=5, \Delta(G)=12$ 
$k$-list-critical graph is one for which there is a $k$-list assignment $\mathcal{L}$ such that the graph is not $\mathcal{L}$-choosable but every proper subgraph is. Every $k$-critical graph is thus also $k$-listcritical, but in general there are $k$-list-critical graphs that are not $k$-critical. Furthermore, a $k$-critical graph cannot contain another $k$-critical graph as a proper subgraph, but this statement is not true if we replace " $k$-critical" with " $k$-list-critical", since a graph may be list-critical with respect to a list assignment $\mathcal{L}$ but not $\mathcal{L}^{\prime}$, yet it may contain a proper subgraph that is list-critical with respect to $\mathcal{L}^{\prime}$ but not $\mathcal{L}$. So, one defines a minimal $k$-list-critical graph to be one which does not contain a $k$-list-critical graph as a proper subgraph.

Observe that a minimal counterexample to our claim that also has the least number of edges must be a minimal 6-list-critical graph. Postle and Thomas [25] showed in 2018 that there are only finitely many 6-list-critical graphs on any surface. Thus, the claim that every toroidal graph has gap at most 2 needs to be verified against only finitely many potential counterexamples; however, a complete list of 6-list-critical graphs on the torus does not yet exist in the literature (though a complete list of 6-critical graphs was given by Thomassen [31] in 1994). Stiebitz, Tuza and Voigt [29] in 2009 showed that for all $2 \leqslant r \leqslant k$, there is a minimal $k$-list critical graph that is $r$-chromatic, so one also cannot immediately rule out the existence of a minimal 6-list-critical graph on the torus that is 3-chromatic.

We note that computing tight bounds even for triangulations on surfaces of higher values of $g$ appears difficult due to the lack of structural results similar to Altshuler's theorem for the torus (Theorem 16).

\subsection{Analogous results for graphs embeddable in nonorientable surfaces}

As mentioned in Section 1, the restriction to orientable surfaces has only been for convenience, and the results proved in Section 4 also hold over nonorientable surfaces with suitable modifications, which we indicate below.

The Heawood number for nonorientable surfaces is

$$
\widetilde{H}(k):=\left\lfloor\frac{7+\sqrt{1+24 k}}{2}\right\rfloor .
$$

By essentially the same argument as given by Heawood [16] for the orientable case, $\widetilde{H}(k)$ is an upper bound for the chromatic number of any graph $G$ embeddable in $N_{k}, k \geqslant 1$, and the argument also carries forward to the choice number, so $\chi(G) \leqslant \chi_{\ell}(G) \leqslant \widetilde{H}(k)$ for every $G$ embeddable in $N_{k}, k \geqslant 1$.

This upper bound is not tight for the Klein bottle $N_{2}$, as shown by Franklin [14] in 1934: $\widetilde{H}(2)=7$, but every graph embeddable in $N_{2}$ is 6-colorable. In particular, $K_{6}$ is embeddable in $N_{2}$, but $K_{7}$ is not. Using Theorem 12 one can show that every graph embeddable in $N_{2}$ is also 6-choosable.

For every other nonorientable surface, Heawood's upper bound is tight. Ringel [26] showed in 1954 that for every $r \geqslant 1$, except $r=2, K_{r}$ is embeddable in $N_{\tilde{\gamma}(r)}$ for $\tilde{\gamma}(r):=\lceil(r-3)(r-4) / 6\rceil$ and this is best possible. This implies that $K_{\widetilde{H}(k)}$ is embeddable 
in $N_{k}$ for all $k \geqslant 1$ except $k=2$. Dirac's map color theorem [1,11] also extends to $N_{k}$ for all $k \geqslant 1$, except $k=2$. In the latter case, $K_{6}$ is not the only 6 -chromatic graph that is embeddable in $N_{2}$ (see [1] for an example of another such graph). Dirac's map color theorem for the choice number $[9,18]$ also extends to $N_{k}$ for all $k \geqslant 1$, except $k=2$.

We define, analogously, the functions $\widetilde{\text { jump }}(k)$ and $\widetilde{\text { jump }}(k, r)$. Using the above results it is not hard to show the following:

Theorem 20. $\widetilde{\text { jump }}(1)=2$. That is, for graphs $G$ embeddable in the projective plane $N_{1}$, $\operatorname{jump}(G) \leqslant 2$. Moreover, this bound is best possible.

The asymptotic bounds on $\widetilde{\text { jump }}(k)$ and $\widetilde{\text { jump }}(k, r)$ are also the same as in the orientable case. The proofs go through in a similar manner. One just needs to know what the result is of adding a handle to a nonorientable surface, for which the following theorem is useful:

Theorem 21 (Dyck [12], 1888). The connected sum of a torus and a projective plane is isomorphic to the connected sum of three projective planes.

One also needs to modify Euler's formula for nonorientable surfaces as follows: if $G$ is embeddable in $N_{k}, k \geqslant 1$, and $\mathrm{v}$, e and $\mathrm{f}$ denote the number of vertices, edges, and faces of $G$ in an embedding of $G$ in $N_{k}$, respectively, then $\mathrm{v}-\mathrm{e}+\mathrm{f} \geqslant 2-k$, with equality holding if every face is homeomorphic to a disc.

\subsection{Concluding remarks}

We have proved in Theorem 3 that any loopless 6-regular triangulation $G$ on the torus satisfies $\operatorname{jump}(G) \leqslant 2$. We speculate that every 3-chromatic toroidal graph is in fact 5-choosable:

Conjecture 22. $\operatorname{jump}(1,3)=2$.

A resolution to Conjecture 22 will also answer Question 2, as we have observed that the maximum gap for toroidal graphs is at least 2, and neither toroidal bipartite graphs nor toroidal graphs with chromatic number at least 4 can attain a gap greater than 2 .

An easy example of a 6-regular triangulation $G$ on the torus for which $\operatorname{jump}(G)=1$ is furnished by $T(3,3,0)$, which is isomorphic to the complete multipartite graph $K_{3 * 3}$. Kierstead [17] in 2000 showed that $\chi_{\ell}\left(K_{3 * r}\right)=\lceil(4 r-1) / 3\rceil$ for every $r \geqslant 1$, so $\chi_{\ell}\left(K_{3 * 3}\right)=$ 4 , and since $T(3,3,0) \equiv T(r, s, t)$ satisfies $s \equiv 0 \equiv r-t(\bmod 3)$, it is 3 -chromatic. Hence, $\operatorname{jump}(T(3,3,0))=1$.

We are also able to show that if $G$ is any 3-chromatic 6-regular toroidal triangulation, then $G$ is not 3 -choosable (except possibly when $G=T(3,9,3)$ ), which will appear in a forthcoming paper [8]. But, we do not have an explicit example of a 6-regular toroidal triangulation for which $\operatorname{jump}(G)=2$. One of the authors is unsure whether any such graph exists, while the other believes that there might be one; in any case, we pose the following question:

Question 23. Are there 3-chromatic 6-regular toroidal triangulations that are 5-listchromatic? 
The results on the asymptotic behavior of jump $(g)$ and jump $(g, r)$ motivate the following conjectures that refine the results proved in Section 4:

Conjecture 24. jump $(g, r)$ is unimodal in $r$ for each fixed $g$. That is, there exists $r_{0} \equiv$ $r_{0}(g)$ such that

$$
\operatorname{jump}(g, 1) \leqslant \operatorname{jump}(g, 2) \leqslant \cdots \leqslant \operatorname{jump}\left(g, r_{0}\right) \geqslant \cdots \geqslant \operatorname{jump}(g, H(g)) .
$$

This is already seen to be true for planar and toroidal graphs. Firstly, a loopless graph is 1-chromatic if and only if it is empty, which implies that it is also 1-list-chromatic, so $\operatorname{jump}(g, 1)=0$ for all $g \geqslant 0$. Next, the results mentioned in Section 1 show that $\operatorname{jump}(0,2)=1, \operatorname{jump}(0,3)=2$, and $\operatorname{jump}(0,4)=1$. Lastly, jump $(0, r)=0$ for $r \geqslant 5$ by the four color theorem due to Appel and Haken [6,7]. So, jump $(0, r)$ is indeed unimodal in $r$.

Similarly, for toroidal graphs, though we don't have precise values of jump $(g, r)$ for all $r$, the discussion so far shows that jump $(1,2)=1$, jump $(1,3)=2$ or 3 , jump $(1,4)=1$ or $2, \operatorname{jump}(1,5)=0$ or $1, \operatorname{jump}(1,6)=0$ and $\operatorname{jump}(1,7)=0$. So, jump $(1, r)$ is unimodal in $r$ as well.

It is not hard to show that unimodality also holds for $\widetilde{\text { jump }}(1, r)$ and $\widetilde{\text { jump }}(2, r)$, that is, for the projective plane and the Klein bottle; so, Conjecture 24 can be extended to the nonorientable case as well.

One also notices that there may be more than one value $r_{0}$ at which the maximum gap is attained. So, define $r_{\max } \equiv r_{\max }(g)$ to be the least value of $r_{0}(g)$ in Conjecture 24 . We conjecture that $\sqrt{g}$ is the correct order of $r$ at which jump $(g, r)$ attains its maximum value for each fixed $g$ :

Conjecture 25. For all sufficiently large $g, r_{\max }=\Theta(\sqrt{g})$.

Again, Conjecture 25 can be extended analogously to the nonorientable case as well.

Finally, a structural result on the Klein bottle $N_{2}$ for 6-regular triangulations, similar in spirit to Altshuler's theorem on the torus (Theorem 16), was given independently by Negami [22] in 1984 and Thomassen [30] in 1991. We pose the following question on the Klein bottle analogous to Question 2 for the torus, and also ask whether the 6-regular triangulations on the Klein bottle can be examined to get a result analogous to Theorem 3 .

Question 26. What is $\widetilde{\text { jump }}(1)$ ? That is, how large can the gap between the choice number and chromatic number be for a graph embeddable on the Klein bottle?

Just as in the toroidal case, it is not hard to show that the maximum gap cannot be smaller than 2, and we ask how large this gap can get.

Question 27. What is the maximum value of $\widetilde{\text { jump }}(G)$ for any loopless 6-regular triangulation $G$ on the Klein bottle?

However, computing $\widetilde{\text { jump }}(k)$ precisely for higher values of $k$ seems difficult for the same reason as in the orientable case. 
Lastly, the proof of Theorem 4 shows that

$$
c_{1} \sqrt{g} \leqslant \operatorname{jump}(g) \leqslant c_{2} \sqrt{g}
$$

for all sufficiently large $g$, with $c_{1}=2 \times 10^{-113}$ and $c_{2}=7$. It would be interesting to see if this can be refined further:

Question 28. Does the limit

$$
\lim _{g \rightarrow \infty} \frac{\operatorname{jump}(g)}{\sqrt{g}}
$$

exist? If yes, what is its value?

Of course, one can also raise the analogous question for $\widetilde{\text { jump }}(k)$ as well.

\section{Acknowledgements}

We thank an anonymous reviewer for their helpful comments, as well as for suggesting Question 28 to us.

\section{References}

[1] M. O. Albertson and J. P. Hutchinson, The three excluded cases of Dirac's map-color theorem, Ann. New York Acad. Sci. 319 (1979), no. 1, 7-17, https://doi.org/10.1111/j.1749-6632.1979.tb32768.x. MR0556001, Zbl 0489.05023

[2] N. Alon, Choice numbers of graphs: A probabilistic approach, Combin. Probab. Comput. 1 (1992), no. 2, 107-114, https://doi.org/10.1017/S0963548300000122. MR1179241, Zbl 0793.05076

[3] _ Degrees and choice numbers, Random Structures Algorithms 16 (2000), no. 4, 364-368, https://doi .org/10.1002/1098-2418(200007) 16:4<364: :AID-RSA5>3.0.C0;2-0. MR1761581, Zbl 0958.05049

[4] N. Alon and M. Tarsi, Colorings and orientations of graphs, Combinatorica 12 (1992), no. 2, 125-134, https://doi.org/10.1007/BF01204715. MR1179249, Zbl 0756.05049

[5] A. Altshuler, Construction and enumeration of regular maps on the torus, Discrete Math. 4 (1973), no. 3, 201-217, https://doi.org/10.1016/S0012-365X(73)80002-0. MR0321797, Zbl 0253.05117

[6] K. I. Appel and W. Haken, Every planar map is four colorable. Part I: Discharging, Illinois J. Math. 21 (1977), no. 3, 429-490, https://doi.org/10.1215/ijm/1256049011. MR0543792, Zbl 0387.05009 
[7] K. I. Appel, W. Haken, and J. A. Koch, Every planar map is four colorable. Part II: Reducibility, Illinois J. Math. 21 (1977), no. 3, 491-567, https://doi.org/10.1215/ijm/1256049012. MR0543793, Zbl 0387.05010

[8] N. Balachandran and B. Sankarnarayanan, 5-list-coloring toroidal 6-regular triangulations in linear time (2021), submitted for publication, available at arXiv:2106.01634.

[9] T. Böhme, B. Mohar, and M. Stiebitz,

Dirac's map-color theorem for choosability, J. Graph Theory 32 (1999), no. 4, 327-339, https://doi org/10.1002/(SICI) 1097-0118(199912)32:4<327: :AID-JGT2>3. 0 . C0;2-B. MR1722227, Zbl 0941.05025

[10] R. L. Brooks, On colouring the nodes of a network, Proc. Cambridge Philos. Soc. 37 (1941), no. 2, 194-197, https://doi.org/10.1017/S030500410002168X. MR0012236, Zbl 0027.26403

[11] G. A. Dirac, Map-colour theorems, Canad. J. Math. 4 (1952), 480-490, https://doi.org/10.4153/cjm-1952-043-9. MR0050869, Zbl 0047.42203

[12] W. Dyck [von Dyck], Beiträge zur Analysis situs. I. Aufsatz: Ein- und zweidimensionale Mannigfaltigkeiten, Math. Ann. 32 (1888), no. 4, 457-512, https://doi.org/10.1007/BF01443580 (German). MR1510522, JFM 20.0519.04

[13] P. Erdös [Erdős], A. L. Rubin, and H. Taylor, Choosability in graphs, Proceedings of the West Coast Conference on Combinatorics, Graph Theory and Computing: Humboldt State University, Arcata, California, September 5-7, 1979 (P. Z. Chinn and D. McCarthy, eds.), Congr. Numer., vol. 26, Util. Math. Publ. Inc., 1980, pp. 125-157, available at https://old.renyi.hu/ p_erdos/1980-07.pdf. MR0593902, Zbl 0469.05032

[14] P. Franklin, A six color problem, J. Math. Phys. 13 (1934), no. 1-4, 363-369, https://doi.org/10.1002/sapm1934131363. Zbl 0010.27502, JFM 60.0501.04

[15] S. Gutner, The complexity of planar graph choosability, Discrete Math. 159 (1996), no. 1-3, 119-130, https://doi.org/10.1016/0012-365X(95)00104-5. MR1415287, Zbl 0865.05066

[16] P. J. Heawood, Map-colour theorem, Quart. J. Pure Appl. Math. 24 (1890), no. 96, 332-338, available at http://resolver.sub.uni-goettingen.de/purl?PPN600494829_0024. JFM 22.0562.02

[17] H. A. Kierstead, On the choosability of complete multipartite graphs with part size three, Discrete Math. 211 (2000), no. 1-3, 255-259, https://doi.org/10.1016/S0012-365X(99)00157-0. MR1735336, Zbl 0944.05031

[18] D. Král' and R. Škrekovski, The last excluded case of Dirac's map-color theorem for choosability, J. Graph Theory 51 (2006), no. 4, 319-354, https://doi.org/10.1002/jgt.20136. MR2207576, Zbl 1094.05026 
[19] M. Mirzakhani, A small non-4-choosable planar graph, Bull. Inst. Combin. Appl. 17 (1996), 15-18. MR1386951, Zbl 0860.05029

[20] A. Nakamoto, K. Noguchi, and K. Ozeki, Extension to 3-colorable triangulations, SIAM J. Discrete Math. 33 (2019), no. 3, 1390-1414, https://doi.org/10.1137/17M1135505. MR3986554, Zbl 1419.05078

[21] S. Negami, Uniqueness and faithfulness of embedding of toroidal graphs, Discrete Math. 44 (1983), no. 2, 161-180, https://doi .org/10.1016/0012-365X (83) 90057-2. MR0689809, Zbl 0508.05033

[22] _ Classification of 6-regular Klein-bottlal graphs, Res. Rep. Inf. Sci. T.I.T. A 96 (1984).

[23] J. A. Noel, B. A. Reed, and H. Wu, A proof of a conjecture of Ohba, J. Graph Theory 79 (2015), no. 2, 86-102, https://doi.org/10.1002/jgt.21819, available at arXiv:1211.1999. MR3338124, Zbl 1320.05045

[24] K. Ohba, On chromatic-choosable graphs, J. Graph Theory 40 (2002), no. 2, 130-135, https://doi.org/10.1002/jgt.10033. MR1899118, Zbl 1004.05030

[25] L. Postle and R. Thomas, Hyperbolic families and coloring graphs on surfaces, Trans. Amer. Math. Soc. Ser. B 5 (2018), 167-221, https://doi.org/10.1090/btran/26, available at arXiv:1609.06749. MR3882883, Zbl 1401.05126

[26] G. Ringel, Bestimmung der Maximalzahl der Nachbargebiete auf nichtorientierbaren Flächen, Math. Ann. 127 (1954), no. 1, 181-214, https://doi.org/10.1007/BF01361120 (German). MR0063011, Zbl 0055.17001

[27] _ Das Geschlecht des vollständigen paaren Graphen, Abh. Math. Sem. Univ. Hambg. 28 (1965), no. 3-4, 139-150, https://doi.org/10.1007/BF02993245 (German). MR0189012, Zbl 0132.21203

[28] G. Ringel and J. W. T. Youngs, Solution of the Heawood map-coloring problem, Proc. Natl. Acad. Sci. USA 60 (1968), no. 2, 438-445, https://doi.org/10.1073/pnas.60.2.438. MR0228378, Zbl 0155.51201

[29] M. Stiebitz, Z. Tuza, and M. Voigt, On list critical graphs, Discrete Math. 309 (2009), no. 15, 4931-4941, https://doi.org/10.1016/j.disc.2008.05.021. MR2531640, Zbl 1229.05157

[30] C. Thomassen, Tilings of the torus and the Klein bottle and vertex-transitive graphs on a fixed surface, Trans. Amer. Math. Soc. 323 (1991), no. 2, 605-635, https://doi.org/10.2307/2001547. MR1040045, Zbl 0722.05031

[31] _ Five-coloring graphs on the torus, J. Combin. Theory Ser. B 62 (1994), no. 1, 11-33, https://doi.org/10.1006/jctb.1994.1052. MR1290628, Zbl 0805.05022 
[32] _ Every planar graph is 5-choosable, J. Combin. Theory Ser. B 62 (1994), no. 1, 180-181, https://doi.org/10.1006/jctb.1994.1062. MR1290638, Zbl 0805.05023

[33] V. G. Vizing, Färbung der Ecken eines Graphen mit vorgeschriebenen Farben [Coloring the vertices of a graph in prescribed colors], Metody Diskretn. Anal. 29 (1976), 3-10 (Russian). MR0498216, Zbl 0362.05060

[34] M. Voigt, List colourings of planar graphs, Discrete Math. 120 (1993), no. 1-3, 215-219, https://doi.org/10.1016/0012-365X(93)90579-I. MR1235909, Zbl 0790.05030

[35] M. Voigt and B. Wirth, On

3-colorable non-4-choosable planar graphs, J. Graph Theory 24 (1997), no. 3, 233-235, https://doi .org/10.1002/(SICI) 1097-0118(199703) 24:3<233: :AID-JGT4>3 . 3. C0;2-C. MR1431668, Zbl 0868.05025 